TRANSACTIONS OF THE

AMERICAN MATHEMATICAL SOCIETY

Volume 362, Number 7, July 2010, Pages 3677-3690

S 0002-9947(10)04949-4

Article electronically published on February 8, 2010

\title{
PICARD GROUPS IN TRIANGULAR GEOMETRY AND APPLICATIONS TO MODULAR REPRESENTATION THEORY
}

\author{
PAUL BALMER
}

\begin{abstract}
For a tensor triangulated $\mathbb{Z} / p$-category $\mathcal{K}$, with spectrum $\operatorname{Spc}(\mathcal{K})$, we construct an injective group homomorphism $\check{\mathrm{H}}^{1}\left(\operatorname{Spc}(\mathcal{K}), \mathbb{G}_{\mathrm{m}}\right) \otimes \mathbb{Z}[1 / p] \hookrightarrow$ $\operatorname{Pic}(\mathcal{K}) \otimes \mathbb{Z}[1 / p]$, where $\operatorname{Pic}(\mathcal{K})$ is the group of $\otimes$-invertible objects of $\mathcal{K}$. In modular representation theory, we prove that this homomorphism induces a rational isomorphism between the Picard group of the projective support variety and the group of endotrivial representations.
\end{abstract}

\section{INTRODUCTION}

The final goal of this article is to prove Theorem 4.7 which establishes the following link between algebraic geometry and modular representation theory.

Theorem. Let $G$ be a finite group and let $k$ be a field of characteristic $p>0$. Let $T(G)$ be the group of endotrivial $k G$-modules and $\mathcal{V}_{G}=\operatorname{Proj}\left(\mathrm{H}^{\bullet}(G, k)\right)$ be the projective support variety of $G$ over $k$. Then gluing induces an isomorphism

$$
\operatorname{Pic}\left(\mathcal{V}_{G}\right) \otimes_{\mathbb{Z}} \mathbb{Q} \stackrel{\sim}{\longrightarrow} T(G) \otimes_{\mathbb{Z}} \mathbb{Q} .
$$

Moreover, this isomorphism maps $\mathcal{O}_{\mathcal{V}_{G}}(d) \otimes \frac{1}{d}$ to the $k G$-module $\Sigma^{1}(k)=\Omega^{-1}(k)$, for any $d>0$ such that $\mathrm{H}^{d}(G, k)$ contains a system of parameters (see Example 4.5).

There is a rich literature on endotrivial modules, justified by their fundamental role in modular representation theory. For instance, Carlson and Thévenaz recently classified them over $p$-groups in [15, 16]. It is worth mentioning that the rank of $T(G)$, i.e. the dimension of the right-hand $\mathbb{Q}$-vector space $T(G) \otimes \mathbb{Q}$ in $(\mathbb{1})$, has been described by Alperin [1; see also Carlson [12] and Balmer-Benson-Carlson [6. Let us indicate to the non-expert reader that methods of algebraic geometry, and in particular support varieties, are extensively used in modular representation theory, including for the study of $T(G)$. Despite all this, the above strong connection (11) between line bundles on $\mathcal{V}_{G}$ and endotrivial $k G$-modules seems to be new.

Beyond the isomorphism (11) itself, our technique for constructing it reaches much further than modular representation theory. It relies on the concept of gluing, which is very standard in algebraic geometry and which inspired the gluing construction of Balmer-Favi [7] in tensor triangular geometry.

Received by the editors June 23, 2008.

2000 Mathematics Subject Classification. Primary 18E30, 20C20.

Key words and phrases. Picard group, gluing, support variety, triangulated category.

The author's research was supported by NSF grant 0654397 .

(C)2010 by Paul Balmer 
Here, "tensor triangular geometry" refers to the study of tensor triangulated categories $\mathcal{K}$ by geometric means. Initiated in [3], this theory got its proper foundation in [4] with the construction of $\operatorname{Spc}(\mathcal{K})$, the spectrum of $\mathcal{K}$, which is the universal topological space in which one can define reasonable closed supports $\operatorname{supp}(a) \subset \operatorname{Spc}(\mathcal{K})$ for all objects $a \in \mathcal{K}$; see Definition 1.3. This space $\operatorname{Spc}(\mathcal{K})$ can be equipped with a sheaf of commutative rings $\mathcal{O}_{\mathcal{K}}$ making it a locally ringed space

$$
\operatorname{Spec}(\mathcal{K})=\left(\operatorname{Spc}(\mathcal{K}), \mathcal{O}_{\mathcal{K}}\right) .
$$

In algebraic geometry, a quasi-compact and quasi-separated scheme $X$ (e.g. a variety) can be reconstructed as the spectrum of its $\otimes$-triangulated category of perfect complexes: $\operatorname{Spec}\left(\mathrm{D}^{\text {perf }}(X)\right) \cong X$; see [4, Thm.6.3(a)] and [10, Thm. 9.5].

Modular representation theory studies representations of a finite group $G$ over a field $k$ for which $k G$ is not semisimple, meaning that there are non-projective $k G$-modules (this is well known to happen exactly when $\operatorname{char}(k)$ divides the order of $G$ ). In this framework, there is a natural tensor triangulated category to consider, namely the stable module category

$$
k G-\mathrm{stab}=\frac{k G-\bmod }{k G-\operatorname{proj}} \quad \text { (quotient as additive categories) }
$$

which exactly measures the non-semisimplicity of $k G$. Remarkably, in this case, the spectrum is isomorphic to the projective support variety: $\operatorname{Spec}(k G-\operatorname{stab}) \cong \mathcal{V}_{G} \stackrel{\text { def }}{=}$ $\operatorname{Proj}\left(\mathrm{H}^{\bullet}(G, k)\right)$; see [4, Thm. 6.3 (b)]. This also holds for finite group schemes.

Other areas of mathematics are involved in tensor triangular geometry, i.e. produce important tensor triangulated categories, including stable homotopy theory, non-commutative geometry, motivic theory, and more. In general though, the spectrum $\operatorname{Spec}(\mathcal{K})$ is simply a locally ringed space, not necessarily a scheme.

The first construction of this paper (Construction 2.6) is a fraction of homomorphisms

$$
\check{\mathrm{H}}^{1}\left(\operatorname{Spc}(\mathcal{K}), \mathbb{G}_{\mathrm{m}}\right) \stackrel{\alpha}{\longleftarrow} \operatorname{Pic}_{\text {loc.tr. }}(\mathcal{K}) \longleftrightarrow \operatorname{Pic}(\mathcal{K})
$$

for any tensor triangulated category $\mathcal{K}$, where $\operatorname{Pic}(\mathcal{K})$ is the group of isomorphism classes of $\otimes$-invertible objects of $\mathcal{K}$, where $\operatorname{Pic}_{\text {loc.tr. }}(\mathcal{K})$ is the subgroup of locally trivial objects and finally, where $\check{\mathrm{H}}^{1}\left(\operatorname{Spc}(\mathcal{K}), \mathbb{G}_{\mathrm{m}}\right)$ denotes the first Čech cohomology group of $\operatorname{Spc}(\mathcal{K})$ with coefficients in the presheaf of units (see Construction 1.12 and Remark 2.5). The main result of the paper, Theorem 3.9, says that for $\mathcal{K}$ in characteristic $p$, the map $\alpha$ is an isomorphism after inverting $p$. Its inverse is obtained via the gluing construction of [7]. Putting things together we get the general result:

Theorem. Suppose that $\mathcal{K}$ is a tensor triangulated $\mathbb{Z} / p$-category for some prime $p$. Suppose $\mathcal{K}$ is rigid (Definition 1.4) and idempotent complete (Definition 1.5), which are mild assumptions (Remark 1.6). Then there exists a well-defined natural monomorphism

$$
\beta: \check{\mathrm{H}}^{1}\left(\operatorname{Spc}(\mathcal{K}), \mathbb{G}_{\mathrm{m}}\right) \otimes_{\mathbb{Z}} \mathbb{Z}[1 / p] \hookrightarrow \operatorname{Pic}(\mathcal{K}) \otimes_{\mathbb{Z}} \mathbb{Z}[1 / p] .
$$

The article is organized as follows. Section 1 is a review of basic tensor triangular geometry. Section 2 reminds the reader of Picard groups, $\mathbb{G}_{\mathrm{m}}$-cocycles and the gluing technique. Section 3 is devoted to the proof of the above general theorem, which we apply to modular representation theory in Section 4. Observe that the 
announced isomorphism $\operatorname{Pic}\left(\mathcal{V}_{G}\right) \otimes \mathbb{Q} \stackrel{\sim}{\rightarrow} T(G) \otimes \mathbb{Q}$ of $(\mathbb{1})$ is an application of the triangular methods in the strong sense: The statement does not involve triangulated categories, whereas the proof does. Finally, this result cannot hold integrally, as we show in Example 4.9 for the quaternion group $Q_{8}$.

\section{Elements of tensor triangular GeOMEtry}

Definition 1.1. A tensor triangulated category $(\mathcal{K}, \otimes, \mathbb{1})$ is a triangulated category $\mathcal{K}$ (see Verdier [23, §II.1]) equipped with a symmetric monoidal structure (see Mac Lane [20, $\S$ XI.1]) $\otimes: \mathcal{K} \times \mathcal{K} \longrightarrow \mathcal{K}$ with unit $\mathbb{1} \in \mathcal{K}$. The tensor $\otimes$ is also assumed exact in each variable. See more in Keller-Neeman [19].

Examples 1.2. Here are two important examples (details can be found in [5, $\S 4]$ ):

(a) For $X$ a quasi-compact and quasi-separated scheme, e.g. $X$ (topologically) noetherian, take $\mathcal{K}=\mathrm{D}^{\text {perf }}(X)$.

(b) For $G$ a finite group and $k$ a field, take $\mathcal{K}=\mathrm{D}^{\mathrm{b}}(k G-\bmod )$ or its quotient $\mathrm{D}^{\mathrm{b}}(k G-\bmod ) / \mathrm{D}^{\mathrm{b}}(k G-$ proj $) \cong k G-$ stab. See more in Section 4 below.

Definition 1.3. In [4, we define the spectrum, $\operatorname{Spc}(\mathcal{K})$, of a tensor triangulated category $\mathcal{K}$ as the set of proper thick triangulated $\otimes$-ideals $\mathcal{P} \subsetneq \mathcal{K}$ which are prime, i.e. such that $a \otimes b \in \mathcal{P}$ implies $a \in \mathcal{P}$ or $b \in \mathcal{P}$. We define for every object $a \in \mathcal{K}$ an open subset $U(a):=\{\mathcal{P} \in \operatorname{Spc}(\mathcal{K}) \mid a \in \mathcal{P}\}$. These form a basis of the topology on $\operatorname{Spc}(\mathcal{K})$. This space is quasi-compact and quasi-separated, i.e. it has a basis of quasi-compact open subsets, namely precisely $\{U(a)\}_{a \in \mathcal{K}}$; see [4, Prop. 2.14(a)]. The support of an object $a \in \mathcal{K}$ is the closed complement of $U(a)$ :

$$
\operatorname{supp}(a):=\{\mathcal{P} \in \operatorname{Spc}(\mathcal{K}) \mid a \notin \mathcal{P}\} \subset \operatorname{Spc}(\mathcal{K}) .
$$

One has $\operatorname{supp}(0)=\varnothing, \operatorname{supp}(\mathbb{1})=\operatorname{Spc}(\mathcal{K}), \operatorname{supp}(a \oplus b)=\operatorname{supp}(a) \cup \operatorname{supp}(b)$, $\operatorname{supp}(\Sigma a)=\operatorname{supp}(a)$ for $\Sigma: \mathcal{K} \rightarrow \mathcal{K}$ the suspension, $\operatorname{supp}(c) \subset \operatorname{supp}(a) \cup \operatorname{supp}(b)$ for every exact triangle $a \rightarrow b \rightarrow c \rightarrow \Sigma a$ and $\operatorname{supp}(a \otimes b)=\operatorname{supp}(a) \cap \operatorname{supp}(b)$.

Definition 1.4. We say that the tensor triangulated category is rigid (in [5] and in [7, we said strongly closed) if there is a functor $D: \mathcal{K}^{\mathrm{op}} \rightarrow \mathcal{K}$ such that

$$
\operatorname{Hom}_{\mathcal{K}}(a \otimes b, c) \simeq \operatorname{Hom}_{\mathcal{K}}(a, D(b) \otimes c) .
$$

Definition 1.5. We say that an additive category $\mathcal{K}$ is idempotent complete if for any idempotent $e=e^{2}: a \rightarrow a$ in $\mathcal{K}$ there exists a decomposition of the object $a=a_{1} \oplus a_{2}$ such that $e=\left(\begin{array}{ll}1 & 0 \\ 0 & 0\end{array}\right)$. It is easy to idempotent complete any additive category $\mathcal{K} \stackrel{\iota}{\hookrightarrow} \tilde{\mathcal{K}}$, and $\tilde{\mathcal{K}}$ is a (rigid) tensor triangulated category if $\mathcal{K}$ is; see [8].

Remark 1.6. Assuming that $\mathcal{K}$ is rigid and idempotent complete is a rather mild condition for applications: It usually holds for $\mathcal{K}$ the category of compact objects in big enough $\otimes$-triangulated categories; see [5. Rem. 2.2]. These assumptions are used for the following general version of a theorem of Carlson in modular representation theory.

Theorem 1.7 ([5, Thm. 2.11]). Let $\mathcal{K}$ be a rigid, idempotent complete, tensor triangulated category. Let $a \in \mathcal{K}$ be an object such that $\operatorname{supp}(a)=Y_{1} \cup Y_{2}$ with $Y_{1}$ and $Y_{2}$ disjoint closed subsets with quasi-compact open complements $\operatorname{Spc}(\mathcal{K}) \backslash Y_{i}$. Then there exists a decomposition $a \cong a_{1} \oplus a_{2}$ with $\operatorname{supp}\left(a_{i}\right)=Y_{i}$ for $i=1,2$. 
Notation 1.8. The commutative ring $\operatorname{End}_{\mathcal{K}}(\mathbb{1})$ acts centrally on any morphism of $\mathcal{K}$ as follows. Let $f: \mathbb{1} \rightarrow \mathbb{1}$ and let $\varphi: a \rightarrow b$ in $\mathcal{K}$; one can define $f \cdot \varphi: a \rightarrow b$ to be the composition from left to right in the commutative diagram

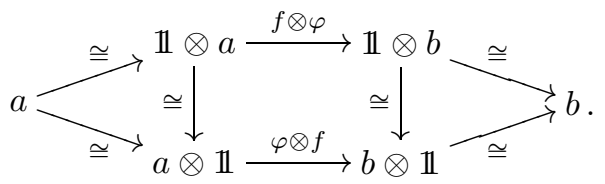

The left and right triangles commute by axioms of symmetric monoidal categories [20, XI.1.(4), p.252], and the middle square by naturality of the switch isomorphism. Plugging $a=b=\mathbb{1}$ in (3) shows that $f \cdot g$ and $f \circ g$ agree on $\operatorname{End}_{\mathcal{K}}(\mathbb{1})$ and also that $\operatorname{End}_{\mathcal{K}}(\mathbb{1})$ is commutative. It follows from the axioms of

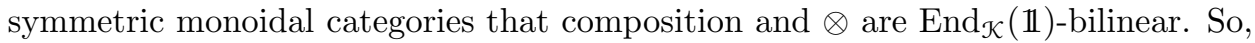
for two morphisms $\varphi_{i}: a_{i} \rightarrow b_{i}, i=1,2$, and for $f \in \operatorname{End}_{\mathcal{K}}(\mathbb{1})$, we have

$$
\left(f \cdot \varphi_{1}\right) \otimes \varphi_{2}=f \cdot\left(\varphi_{1} \otimes \varphi_{2}\right)=\varphi_{1} \otimes\left(f \cdot \varphi_{2}\right): \quad a_{1} \otimes a_{2} \longrightarrow b_{1} \otimes b_{2} .
$$

Construction 1.9. Recall the notion of Verdier localization of triangulated categories; see [23, $§$ II.2]. Consider a thick triangulated subcategory $\mathcal{J} \subset \mathcal{K}$. "Thick" means that for $a, b \in \mathcal{K}$, if $a \oplus b \in \mathcal{J}$, then $a, b \in \mathcal{J}$. Then $\mathcal{K} / \mathcal{J}$ has the same objects as $\mathcal{K}$ but new morphisms $\operatorname{Hom}_{\mathcal{K} / \mathcal{J}}(a, b)$ given by equivalence classes of fractions

$$
a \stackrel{s}{\longleftarrow} \stackrel{f}{\longrightarrow} b,
$$

where cone $(s) \in \mathcal{J}$ and where two fractions are equivalent if they admit a common amplification. When $\mathcal{K}$ is a (rigid) tensor triangulated category and $\mathcal{J}$ is a $\otimes$-ideal, $\mathcal{K} / \mathcal{J}$ inherits an obvious structure of (rigid) tensor triangulated category, such that the localization functor $q: \mathcal{K} \rightarrow \mathcal{K} / \mathcal{J}$ is $\otimes$-triangulated; see [5, Prop. 2.15 (ii)]. For us, the important case of Verdier localization occurs when $\mathcal{J}$ is equal to

$$
\mathcal{K}_{Z}:=\{a \in \mathcal{K} \mid \operatorname{supp}(a) \subset Z\}
$$

for a closed $Z \subset \operatorname{Spc}(\mathcal{K})$, assuming that its open complement $U:=\operatorname{Spc}(\mathcal{K}) \backslash Z$ is quasi-compact. We then define $\mathcal{K}(U)$ to be the idempotent completion of the corresponding Verdier localization. We have the following commutative diagram, defining $\operatorname{res}_{U}$ :

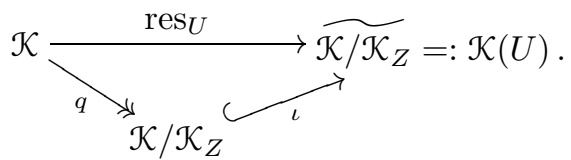

The importance of the idempotent completion comes from Theorem 1.7 that we need to apply to $\mathcal{K}(U)$ as well. Indeed $\mathcal{K}(U)$ is still a tensor triangulated category with $\mathbb{1}_{\mathcal{K}(U)}=\operatorname{res}_{U}\left(\mathbb{1}_{\mathcal{K}}\right)$ and its spectrum is $\operatorname{Spc}(\mathcal{K}(U))=U$; see [7, Prop. 1.11].

Remark 1.10. This definition of $\mathcal{K}(U)$ originates in Thomason's theorem which says that for a quasi-compact open subset $U \subset X$ of a quasi-compact and quasiseparated scheme $X$, the above construction (5) applied to $\mathcal{K}=\mathrm{D}^{\text {perf }}(X)$ gives $\mathcal{K}(U)=\mathrm{D}^{\text {perf }}(U)$; see Thomason-Trobaugh $[22, \S 5.2]$, as also explained in [3, Thm. 2.13].

Convention 1.11. For a quasi-compact open $U \subset \operatorname{Spc}(\mathcal{K})$, we use the shorthand "on $U$ " to mean "in $\mathcal{K}(U)$, after applying the appropriate functor $\operatorname{res}_{U}$ ". 
Construction 1.12. Construction 1.9 can be used to define a presheaf of commutative rings $\mathrm{p}_{\mathcal{K}}$ on $\operatorname{Spc}(\mathcal{K})$, setting for every quasi-compact open $U \subset \operatorname{Spc}(\mathcal{K})$

$$
{ }_{\mathrm{p}} \mathcal{O}_{\mathcal{K}}(U):=\operatorname{End}_{\mathcal{K}(U)}(\mathbb{1}) .
$$

The sheafification of $\mathcal{O}_{\mathcal{K}}$ defines the structure sheaf $\mathcal{O}_{\mathcal{K}}$ and gives us the ringed space $\operatorname{Spec}(\mathcal{K}):=\left(\operatorname{Spc}(\mathcal{K}), \mathcal{O}_{\mathcal{K}}\right)$. There is a sub-presheaf $\mathbb{G}_{\mathrm{m}}$ of ${ }_{\mathrm{p}} \mathcal{O}_{\mathcal{K}}$ defined as the automorphisms of the unit:

$$
\mathrm{p}_{\mathbb{G}_{\mathrm{m}}}(U):=\operatorname{Aut}_{\mathcal{K}(U)}(\mathbb{1}) \subset \mathrm{p}_{\mathcal{K}}(U)
$$

for $U \subset \operatorname{Spc}(\mathcal{K})$ quasi-compact open.

\section{INVERTIBLE OBJECTS AND COCYCLES}

As above, $\mathcal{K}$ is a tensor triangulated category, assumed rigid (Definition 1.4) and idempotent complete (Definition 1.5). Recall Convention 1.11.

Definition 2.1. An object $x \in \mathcal{K}$ is $\otimes$-invertible if there exists $y \in \mathcal{K}$ such that $x \otimes y \simeq \mathbb{1}$. Since $\mathcal{K}$ is rigid, this is equivalent to the obvious (evaluation) map $x \otimes D(x) \rightarrow \mathbb{1}$ being an isomorphism, i.e. we have $y \simeq D(x)$. We denote by $\operatorname{Pic}(\mathcal{K})$ the abelian group of isomorphism classes $[x]$ of $\otimes$-invertible objects, with addition $[x]+[y]=[x \otimes y]$ and zero $0=[\mathbb{1}]$.

Remark 2.2. An object $x \in \mathcal{K}$ which is locally $\otimes$-invertible, in the sense that $\operatorname{Spc}(\mathcal{K})$ can be covered by open subsets $U$ such that $x$ is $\otimes$-invertible on $U$, is necessarily $\otimes$-invertible in $\mathcal{K}$. Indeed, the natural map $x \otimes D(x) \rightarrow \mathbb{1}$ is a local isomorphism, hence an isomorphism; see [7, Lem. 6.2].

Definition 2.3. We say that a $\otimes$-invertible object $x \in \mathcal{K}$ is locally trivial if $\operatorname{Spc}(\mathcal{K})$ can be covered by quasi-compact open subsets $U$ such that $x \simeq \mathbb{1}$ on $U$. (We call such a cover trivializing.) This defines a subgroup of $\operatorname{Pic}(\mathcal{K})$, that we denote as

$$
\operatorname{Pic}_{\text {loc.tr. }}(\mathcal{K}):=\{[x] \in \operatorname{Pic}(\mathcal{K}) \mid x \text { is locally trivial }\} \subset \operatorname{Pic}(\mathcal{K}) .
$$

Example 2.4. For $X$ a scheme, we have $\operatorname{Pic}_{\text {loc.tr. }}\left(D^{\text {perf }}(X)\right)=\operatorname{Pic}(X)$. Indeed, $\operatorname{Pic}\left(\mathrm{D}^{\text {perf }}(X)\right)=\operatorname{Pic}(X) \oplus \mathbb{Z}^{m}$, where $m$ is the number of connected components of $X$ and where $\mathbb{Z}^{m}$ comes from shifting complexes; see [7, Prop. 6.4].

Remark 2.5. We now use Čech cohomology of the topological space $\operatorname{Spc}(\mathcal{K})$ with coefficients in the presheaf $\mathbb{p}_{\mathrm{m}}$; see Construction 1.12. We only use the first group $\check{\mathrm{H}}^{1}\left(\operatorname{Spc}(\mathcal{K}), \mathrm{p} \mathbb{G}_{\mathrm{m}}\right)$, so let us specifically recall that one. Since the space $\operatorname{Spc}(\mathcal{K})$ is quasi-separated (see Definition 1.3), we only consider finite covers by quasi-compact opens $\operatorname{Spc}(\mathcal{K})=U_{1} \cup \cdots \cup U_{n}$. As always, we write

$$
U_{i_{1} i_{2} \ldots i_{r}}:=U_{i_{1}} \cap U_{i_{2}} \cap \cdots \cap U_{i_{r}} \quad \text { for any } \quad 1 \leq i_{1}, \ldots, i_{r} \leq n .
$$

A "1-cocycle" for $\mathrm{p} \mathbb{G}_{\mathrm{m}}$ over $U_{*}$ is the data of $\sigma_{j i} \in \mathrm{p} \mathbb{G}_{\mathrm{m}}\left(U_{i j}\right)$ for all $1 \leq i, j \leq n$ satisfying the condition $\sigma_{k j} \cdot \sigma_{j i}=\sigma_{k i}$ in $\mathbb{p}_{\mathrm{m}}\left(U_{i j k}\right)$. These cocycles form an abelian group under multiplication. For a collection $\tau_{i} \in \mathrm{p} \mathbb{G}_{\mathrm{m}}\left(U_{i}\right)$ we define the "1boundary" $d \tau$ by $d \tau_{j i}=\tau_{j} \tau_{i}^{-1}$. The quotient of the abelian group of 1-cocycles by the subgroup of 1-boundaries is $\check{\mathrm{H}}^{1}\left(U_{*}, \mathrm{p} \mathbb{G}_{\mathrm{m}}\right)$. For any refinement $V_{*}$ of $U_{*}$, there is an obvious map $\check{\mathrm{H}}^{1}\left(U_{*}, \mathrm{p} \mathbb{G}_{\mathrm{m}}\right) \rightarrow \check{\mathrm{H}}^{1}\left(V_{*}, \mathrm{p} \mathbb{G}_{\mathrm{m}}\right)$. One defines the abelian group $\check{\mathrm{H}}^{1}\left(\operatorname{Spc}(\mathcal{K}), \mathbb{G}_{\mathrm{m}}\right)$ as the colimit of those groups. This abuse of notation is justified by the examples, where $\mathrm{p} \mathbb{G}_{\mathrm{m}}$ is a sheaf. 
Construction 2.6. There exists, as usual, a group homomorphism

$$
\alpha: \operatorname{Pic}_{\text {loc.tr. }}(\mathcal{K}) \rightarrow \check{\mathrm{H}}^{1}\left(\operatorname{Spc}(\mathcal{K}), \mathbb{G}_{\mathrm{m}}\right),
$$

natural in $\mathcal{K}$ and defined as follows. For a class $\ell \in \operatorname{Pic}_{\text {loc.tr. }}(\mathcal{K})$, choose some $x \in \ell$. Choose a trivializing cover $U_{*}=\left(U_{i}\right)_{i=1}^{n}$ of $\operatorname{Spc}(\mathcal{K})$ by quasi-compact open subsets and choose isomorphisms $\xi_{i}: x \stackrel{\sim}{\rightarrow} \mathbb{1}$ on $U_{i}$. Define $\sigma=\sigma(x, \xi)$ by $\sigma_{j i}=\xi_{j} \circ \xi_{i}^{-1}$ for all $1 \leq i, j \leq n$. This defines an element $\sigma_{j i} \in \mathrm{p} \mathbb{G}_{\mathrm{m}}\left(U_{i} \cap U_{j}\right)$; see (7). The class of the cocycle $\sigma=\sigma(x, \xi)$ in $\check{\mathrm{H}}^{1}\left(U_{*}, \mathrm{p} \mathbb{G}_{\mathrm{m}}\right)$ is independent of the choice of the isomorphisms $\xi_{i}: x \stackrel{\sim}{\rightarrow} \mathbb{1}$; for other choices $\xi_{i}^{\prime}: x \stackrel{\sim}{\rightarrow} \mathbb{1}$ give $\tau_{i}:=\xi_{i}^{\prime} \xi_{i}^{-1} \in \mathrm{p} \mathbb{G}_{\mathrm{m}}\left(U_{i}\right)$ such that $\sigma\left(x, \xi^{\prime}\right) \cdot \sigma(x, \xi)^{-1}=d \tau$. See Notation 1.8 if necessary. Hence $\left[\sigma\left(x, \xi^{\prime}\right)\right]-[\sigma(x, \xi)]=$ $[d \tau]=0$ in $\check{\mathrm{H}}^{1}\left(U_{*}, \mathrm{p} \mathbb{G}_{\mathrm{m}}\right)$. Similarly, this class $[\sigma(x)] \in \check{\mathrm{H}}^{1}\left(U_{*}, \mathrm{p} \mathbb{G}_{\mathrm{m}}\right)$ is independent of the chosen representative $x \in \ell$. Indeed, for an isomorphism $\varphi: y \stackrel{\sim}{\rightarrow} x$, by the above, one can compute the class of $\sigma(y)$ by using the trivialization $\xi_{*} \circ \varphi$, which gives the same cocycle $\xi_{j} \varphi\left(\xi_{i} \varphi\right)^{-1}=\xi_{j} \xi_{i}^{-1}$. Then $\alpha(\ell) \in \check{\mathrm{H}}^{1}\left(\operatorname{Spc}(\mathcal{K}), \mathbb{G}_{\mathrm{m}}\right)$ is defined as the image of our class $[\sigma] \in \check{\mathrm{H}}^{1}\left(U_{*}, \mathrm{p} \mathbb{G}_{\mathrm{m}}\right)$ in the colimit :

$$
\begin{aligned}
& \check{\mathrm{H}}^{1}\left(U_{*}, \mathrm{p} \mathbb{G}_{\mathrm{m}}\right) \longrightarrow \operatorname{colim}_{U_{*}} \check{\mathrm{H}}^{1}\left(U_{*}, \mathrm{p} \mathbb{G}_{\mathrm{m}}\right)=\check{\mathrm{H}}^{1}\left(\operatorname{Spc}(\mathcal{K}), \mathbb{G}_{\mathrm{m}}\right) \\
& {[\sigma] \quad \longmapsto \quad \alpha(\ell) \text {. }}
\end{aligned}
$$

Sending $[\sigma]$ in the colimit over the open covers $U_{*}$ (under refinement) makes the construction independent of the choice of the trivializing cover $U_{*}$.

As a partial converse to the above, we now recall the gluing technique of [7].

Definition 2.7. A gluing data is a triple $\left(U_{*}, x_{*}, \sigma_{* *}\right)$, where $U_{*}=\left(U_{i}\right)_{i=1}^{n}$ is a finite open cover of the spectrum $\operatorname{Spc}(\mathcal{K})$ by quasi-compact open subsets, where $x_{*}=\left(x_{i}\right)_{i=1}^{n}$ is a collection of objects $x_{i} \in \mathcal{K}\left(U_{i}\right)$ on each $U_{i}$, and finally $\sigma_{* *}=$ $\left(\sigma_{i j}\right)_{1 \leq i, j \leq n}$ is a collection of isomorphisms $\sigma_{j i}: x_{i} \stackrel{\sim}{\rightarrow} x_{j}$ on $U_{i j}$ (see (8) above) satisfying the cocycle condition: $\sigma_{k j} \circ \sigma_{j i}=\sigma_{k i}$ on $U_{i j k}$ for every $1 \leq i, j, k \leq n$.

We call gluing of the data $\left(U_{*}, x_{*}, \sigma_{* *}\right)$ a pair $\left(x, \xi_{*}\right)$ consisting of an object $x \in \mathcal{K}$ and a collection $\xi_{*}=\left(\xi_{i}\right)_{i=1}^{n}$ of isomorphisms $\xi_{i}: x \stackrel{\sim}{\rightarrow} x_{i}$ on $U_{i}$ compatible with $\sigma_{* *}$, i.e. such that $\sigma_{j i} \circ \xi_{i}=\xi_{j}$ on $U_{i j}$ for every $1 \leq i, j \leq n$ :

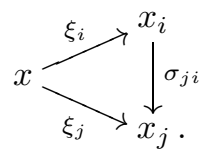

We alternatively say that the gluing data $\left(U_{*}, x_{*}, \sigma_{* *}\right)$ admits a solution $\left(x, \xi_{*}\right)$.

Suppose that $\left(x, \xi_{*}\right)$ and $\left(y, \eta_{*}\right)$ are two gluings for the same data $\left(U_{*}, x_{*}, \sigma_{* *}\right)$. An isomorphism of gluings $f:\left(x, \xi_{*}\right) \stackrel{\sim}{\rightarrow}\left(y, \eta_{*}\right)$ is an isomorphism $f: x \stackrel{\sim}{\rightarrow} y$ in $\mathcal{K}$, such that $\eta_{i} \circ f=\xi_{i}$ on $U_{i}$ for all $1 \leq i \leq n$.

Here, we shall only glue $\otimes$-invertible objects $x_{i}$, mostly $x_{i}=\mathbb{1}$ for all $i$, although we need other $x_{i}$ 's for induction arguments. In this situation, a gluing $x \in \mathcal{K}$ as above must be $\otimes$-invertible in $\mathcal{K}$ as well; see Remark 2.2. Using Theorem 1.7, one can prove:

Theorem 2.8 (Balmer-Favi [7, Cor. 5.10]). Let $\mathcal{K}$ be a rigid (Definition 1.4) and idempotent complete (Definition 1.5) $\otimes$-triangulated category. Then the gluing problem for two open subsets admits a solution, unique up to possibly non-unique isomorphism. 
Remark 2.9. The non-uniqueness of the isomorphism in Theorem 2.8 might sound secondary, but it has consequences for the gluing of three pieces, i.e. when $\operatorname{Spc}(\mathcal{K})=$ $U_{1} \cup U_{2} \cup U_{3}$ and we want to glue three objects. What happens is that the gluing still exists but is no longer unique up to isomorphism. Worst, with four pieces or more, the gluing simply does not exist in general. In the next section, we explain how to treat this problem in positive characteristic.

\section{Gluing in Characteristic $p$, Up to $p$-Th POWERS}

As before, we assume for the whole section that $\mathcal{K}$ is a rigid (Definition 1.4), idempotent complete (Definition [1.5), tensor triangulated category. We further assume that $p=0$ in $\mathcal{K}$ for some prime number $p$; that is, $\mathcal{K}$ is a $\mathbb{Z} / p$-category. This holds of course in modular representation theory, or for $\mathcal{K}=\mathrm{D}^{\text {perf }}(X)$ when $X$ is a scheme over $\mathbb{Z} / p$.

We consider an open cover of the spectrum by quasi-compact open subsets

$$
\operatorname{Spc}(\mathcal{K})=U_{1} \cup \cdots \cup U_{n},
$$

and we denote by $Z_{i}=\operatorname{Spc}(\mathcal{K}) \backslash U_{i}$ their closed complements. Recall Convention 1.11. We start with a result which does not use $p=0$ in $\mathcal{K}$.

Proposition 3.1. If a morphism $f: a \rightarrow b$ in $\mathcal{K}$ vanishes on each $U_{i}$, then $f^{\otimes n}=0$ in $\mathcal{K}$, as a morphism from the object $a^{\otimes n}:=a \otimes \cdots \otimes a$ ( $n$ factors) to $b^{\otimes n}$.

Proof. This follows from [4, Prop. 2.21], but we sketch it for the reader's convenience. Since $\iota: \mathcal{K} / \mathcal{K}_{Z_{i}} \hookrightarrow \mathcal{K}\left(U_{i}\right)$ is fully faithful, we have $f=0$ in $\mathcal{K} / \mathcal{K}_{Z_{i}}$. By a general fact of Verdier localization (see [4, Lem. 2.22] if necessary), $f$ factors via an object $c_{i} \in \mathcal{K}_{Z_{i}}$. Since $\bigcap_{i} Z_{i}=\varnothing$, the product $c_{1} \otimes \cdots \otimes c_{n}$ has empty support, hence vanishes ([5, Cor. 2.5]). So, $f^{\otimes n}$ factors via $c_{1} \otimes \cdots \otimes c_{n}=0$.

Remark 3.2. We cannot deduce that if $f=g$ on each $U_{i}$, then $f^{\otimes r}=g^{\otimes r}$ for $r$ large enough. Set for instance $f=\operatorname{id}_{a \oplus b}$ and $g=\left(\begin{array}{ll}1 & 0 \\ h & 1\end{array}\right)$ for $h: a \rightarrow b$ locally zero but not zero. Then $g^{\otimes r} \neq$ id for all $r \geq 1$. Hence the importance of the next result.

Lemma 3.3. Let $x, y \in \mathcal{K}$ be $\otimes$-invertible objects (Definition 2.1) and let $\varphi, \psi$ : $x \stackrel{\sim}{\rightarrow} y$ be two isomorphisms such that $\varphi=\psi$ on $U_{i}$ for all $1 \leq i \leq n$. Then $\varphi^{\otimes p^{r}}=\psi^{\otimes p^{r}}: x^{\otimes p^{r}} \stackrel{\sim}{\rightarrow} y^{\otimes p^{r}}$ for $r \geq 0$ large enough $\left(p^{r} \geq n\right.$ will do).

Proof. For a $\otimes$-invertible object $y$, the functor $-\otimes y: \mathcal{K} \rightarrow \mathcal{K}$ is an equivalence. We have in particular an isomorphism $\operatorname{End}_{\mathcal{K}}(\mathbb{1}) \stackrel{\sim}{\rightarrow} \operatorname{End}_{\mathcal{K}}(y)$, given by $f \mapsto f \cdot \operatorname{id}_{y}$, using Notation 1.8. Therefore, the automorphism $\varphi \circ \psi^{-1}: y \stackrel{\sim}{\rightarrow} y$ is of the form $f \cdot \operatorname{id}_{y}$ for some $f: \mathbb{1} \stackrel{\sim}{\rightarrow} \mathbb{1}$, that we can write $f=1+h$, if we wish. The hypothesis $\varphi=\psi$ on each $U_{i}$ implies that $h$ is zero on each $U_{i}$. So $h^{\otimes n}=0$ by Proposition 3.1. Now, on the ring $\operatorname{End}_{\mathcal{K}}(\mathbb{1})$, composition and $\otimes$ coincide (see 1.8), hence $h^{n}=0$.

In short, $\varphi=(1+h) \cdot \psi$ for $h \in \operatorname{End}_{\mathcal{K}}(\mathbb{1})$ such that $h^{p^{r}}=0$ for $r \gg 0$. Then

$$
\varphi^{\otimes p^{r}}=((1+h) \cdot \psi)^{\otimes p^{r}} \stackrel{\underline{\underline{(4)}}}{=}(1+h)^{p^{r}} \cdot \psi^{\otimes p^{r}}=\psi^{\otimes p^{r}},
$$

since $(1+h)^{p^{r}}=1+h^{p^{r}}$ as $p=0$ in the $\operatorname{ring} \operatorname{End}_{\mathcal{K}}(\mathbb{1})$, by assumption.

Lemma 3.4. Let $x, y \in \mathcal{K}$ be $\otimes$-invertible objects and let $\varphi_{i}: x \stackrel{\sim}{\rightarrow} y$ be isomorphisms on $U_{i}$ such that $\varphi_{i}=\varphi_{j}$ on $U_{i j}$. Then there exist $r \geq 0$ large enough and an isomorphism $\varphi: x^{\otimes p^{r}} \stackrel{\sim}{\rightarrow} y^{\otimes p^{r}}$ such that $\varphi=\left(\varphi_{i}\right)^{\otimes p^{r}}$ on each $U_{i}$. 
Proof. It is a result of [7] that for a cover by two open subsets $\operatorname{Spc}(\mathcal{K})=U \cup V$ there exists a Mayer-Vietoris long exact sequence, which says in particular that for every object $a, b \in \mathcal{K}$ the following sequence is exact in the middle:

$$
\operatorname{Hom}_{\mathcal{K}}(a, b) \stackrel{\left(\begin{array}{l}
\text { res } \\
\text { res }
\end{array}\right)}{\longrightarrow} \operatorname{Hom}_{\mathcal{K}(U)}(a, b) \oplus \operatorname{Hom}_{\mathcal{K}(V)}(a, b) \stackrel{(- \text { res res })}{\longrightarrow} \operatorname{Hom}_{\mathcal{K}(U \cap V)}(a, b) .
$$

In general, the left-hand map is not injective, but we circumvent this via Lemma 3.3 The proof proceeds by induction on the number $n$ of open subsets in (10). The case $n=1$ is trivial, so let us assume $n \geq 2$. Let $V=U_{2} \cup \cdots \cup U_{n}$. By induction hypothesis, there exist $r \geq 0$, and an isomorphism $\psi: x^{\otimes p^{r}} \stackrel{\sim}{\rightarrow} y^{\otimes p^{r}}$ on $V$ such that $\psi=\varphi_{i}^{\otimes p^{r}}$ on each $U_{i}$, for $2 \leq i \leq n$. We have $U_{1} \cap V=U_{12} \cup \cdots \cup U_{1 n}$ and the morphisms $\varphi_{1}^{\otimes p^{r}}$ and $\psi$ agree on each little piece $U_{1 i}$ for $2 \leq i \leq n$ by construction of $\psi$ and since $\varphi_{1}$ and $\varphi_{i}$ agree on $U_{1 i}$. Applying Lemma 3.3 to the category $\mathcal{K}\left(U_{1} \cap V\right)$, we see that there exists $s \geq 1$ such that $\varphi_{1}^{\otimes p^{r+s}}=\psi^{\otimes p^{s}}$ on $U_{1} \cap V$. By Mayer-Vietoris (11), applied to $U=U_{1}$ and to our $V$, there exists a morphism $\varphi: x^{\otimes p^{r+s}} \rightarrow y^{\otimes p^{r+s}}$ in $\mathcal{K}$ such that $\varphi=\varphi_{1}^{\otimes p^{r+s}}$ on $U_{1}$ and such that $\varphi=\psi^{\otimes p^{s}}$ on $V$, which implies $\varphi=\psi^{\otimes p^{s}}=\varphi_{i}^{\otimes p^{r+s}}$ on each $U_{i}$, for $2 \leq i \leq n$. This morphism $\varphi$ is locally an isomorphism, so it is an isomorphism.

Remark 3.5. Let $\left(U_{*}, x_{*}, \sigma_{* *}\right)$ and $\left(U_{*}, x_{*}^{\prime}, \sigma_{* *}^{\prime}\right)$ be gluing data as in Definition 2.7 for the same open cover $U_{*}$. Then we can define a new gluing data $\left(U_{*}, x_{*} \otimes x_{*}^{\prime}, \sigma_{* *} \otimes \sigma_{* *}^{\prime}\right)$ with objects $x_{i} \otimes x_{i}^{\prime}$ on $U_{i}$ and isomorphisms $\sigma_{j i} \otimes \sigma_{j i}^{\prime}: x_{i} \otimes x_{i}^{\prime} \stackrel{\sim}{\rightarrow} x_{j} \otimes x_{j}^{\prime}$ on $U_{i j}$. Similarly, if $\left(x, \xi_{*}\right)$ and $\left(x^{\prime}, \xi_{*}^{\prime}\right)$ are gluings of the original data, respectively, then $\left(x \otimes x^{\prime}, \xi_{*} \otimes \xi_{*}^{\prime}\right)$ is a gluing of the new one. In particular, for $r \geq 1$, if $\left(x, \xi_{*}\right)$ is a gluing of $\left(U_{*}, x_{*}, \sigma_{* *}\right)$, then $\left(x^{\otimes r}, \xi_{*}^{\otimes r}\right)$ is a gluing of $\left(U_{*}, x_{*}^{\otimes r}, \sigma_{* *}^{\otimes r}\right)$.

Proposition 3.6 (Uniqueness of gluing up to $p$-th powers). Suppose that $\left(x, \xi_{*}\right)$ and $\left(y, \eta_{*}\right)$ are two gluings for the same data $\left(U_{*}, x_{*}, \sigma_{* *}\right)$. Suppose that the objects $x_{i}$ are $\otimes$-invertible in $\mathcal{K}\left(U_{i}\right)$. Then there exist $r \geq 0$ and an isomorphism of gluings $\left(x^{\otimes p^{r}}, \xi_{*}^{\otimes p^{r}}\right) \stackrel{\sim}{\rightarrow}\left(y^{\otimes p^{r}}, \eta_{*}^{\otimes p^{r}}\right)$ for the data $\left(U_{*}, x_{*}^{\otimes p^{r}}, \sigma_{* *}^{\otimes p^{r}}\right)$.

Proof. The isomorphisms $\varphi_{i}:=\eta_{i}^{-1} \circ \xi_{i}: x \stackrel{\sim}{\longrightarrow} y$ on $U_{i}$ agree on $U_{i j}$ since

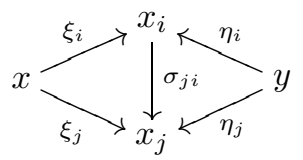

commutes on $U_{i j}$ by definition; see (9). We conclude by Lemma 3.4

Proposition 3.7 (Existence of gluing up to $p$-th powers). Let $\left(U_{*}, x_{*}, \sigma_{* *}\right)$ be a gluing data (Definition 2.7). Suppose that the objects $x_{i}$ are $\otimes$-invertible in $\mathcal{K}\left(U_{i}\right)$. Then there exists $r \geq 0$ such that the data $\left(U_{*}, x_{*}^{\otimes p^{r}}, \sigma_{* *}^{\otimes p^{r}}\right)$ admits a gluing.

Proof. The proof goes by induction on the number $n$ of open subsets in (10) as above. The case $n=1$ is trivial, so let us assume $n \geq 2$. Consider as before $V=U_{2} \cup \cdots \cup U_{n}$ and let $U_{*}^{\prime}=\left(U_{i}\right)_{i=2}^{n}$. By induction hypothesis, there exists $r \geq 0$ such that the data $\left(U_{*}^{\prime}, x_{*}^{\otimes p^{r}}, \sigma_{* *}^{\otimes p^{r}}\right)$ admits a gluing, say $\left(y, \eta_{*}\right)$ on $V$. In order to apply Theorem 2.8 to the cover $\operatorname{Spc}(\mathcal{K})=U_{1} \cup V$ and to $x_{1}^{\otimes p^{r}} \in \mathcal{K}\left(U_{1}\right)$ and $y \in \mathcal{K}(V)$, we would need them to be isomorphic on $U_{1} \cap V$, which fails a priori. Nevertheless, both $\left(x_{1}^{\otimes p^{r}}, \sigma_{* 1}^{\otimes p^{r}}\right)$ and $\left(y, \eta_{*}\right)$ are gluings on $U_{1} \cap V$ for the same 
gluing data $\left(U_{*}^{\prime \prime}, x_{*}^{\otimes p^{r}}, \sigma_{* *}^{\otimes p^{r}}\right)$ where $U_{*}^{\prime \prime}=\left(U_{1 i}\right)_{i=2}^{n}$. By Proposition 3.6 applied to the category $\mathcal{K}\left(U_{1} \cap V\right)$, there exist $s \geq 0$ and an isomorphism $\psi: x_{1}^{\otimes p^{r+s}} \stackrel{\sim}{\rightarrow} y^{\otimes p^{s}}$ on $U_{1} \cap V$ compatible with $\sigma_{i 1}^{\otimes p^{r+s}}$ and $\eta_{i}^{\otimes p^{s}}$ on $U_{1 i}$ for $2 \leq i \leq n$. We conclude by Theorem 2.8 that there exist $x \in \mathcal{K}$ and isomorphisms $\xi_{1}: x \stackrel{\sim}{\rightarrow} x_{1}^{\otimes p^{r+s}}$ on $U_{1}$ and $\xi^{\prime}: x \stackrel{\sim}{\rightarrow} y^{\otimes p^{s}}$ on $V$ compatible with $\psi$ on $U_{1} \cap V$. We deduce an isomorphism $\xi_{i}:=\eta_{i}^{\otimes p^{s}} \circ \operatorname{res}_{U_{i}}\left(\xi^{\prime}\right): x \stackrel{\sim}{\rightarrow} x_{i}^{\otimes p^{r+s}}$ on $U_{i}$ for all $2 \leq i \leq n$. We have the wanted gluing $\left(x, \xi_{*}\right)$ of the data $\left(U_{*}, x_{*}^{\otimes p^{r+s}}, \sigma_{* *}^{\otimes p^{r+s}}\right)$. Indeed, the remaining compatibility between $\xi_{*}$ and $\sigma_{* *}^{\otimes p^{r+s}}$ is visible in the following diagram of isomorphisms :

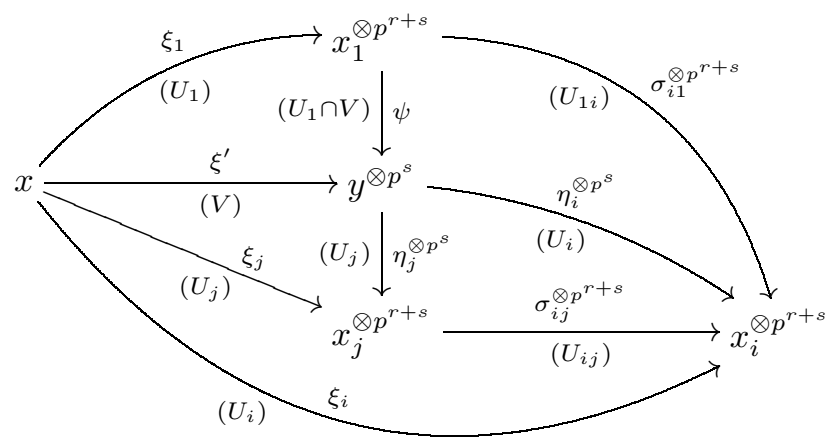

for $2 \leq i, j \leq n$.

Under each isomorphism, we indicate between parentheses on which open it is defined. Each triangle commutes where all its edges are defined.

Proposition 3.8 (Vanishing on boundaries up to $p$-th powers). Suppose that $\left(x, \xi_{*}\right)$ is a gluing of a data $\left(U_{*}, \mathbb{1}_{*}, \sigma_{* *}\right)$, i.e. every $x_{i}=\mathbb{1}$. Suppose that $\left[\sigma_{* *}\right]=0$ in $\check{\mathrm{H}}^{1}\left(U_{*}, \mathrm{p} \mathbb{G}_{\mathrm{m}}\right)$. Then there exist $r \geq 0$ and an isomorphism $x^{\otimes p^{r}} \stackrel{\sim}{\rightarrow} \mathbb{1}$ in $\mathcal{K}$.

Proof. There exists $\tau_{*} \in \operatorname{Aut}_{\mathcal{K}\left(U_{*}\right)}(\mathbb{1})$ such that $\sigma_{j i}=\left(d \tau_{*}\right)_{j i}=\tau_{j} \tau_{i}^{-1}$ on $U_{i j}$ for all $1 \leq i, j \leq n$. Define $\varphi_{i}:=\tau_{i}^{-1} \xi_{i}: x \stackrel{\sim}{\rightarrow} \mathbb{1}$ on each $U_{i}$. The above relation and the compatibility of $\xi_{*}$ with $\sigma_{* *}$ in (9) imply that $\varphi_{i}=\varphi_{j}$ on $U_{i j}$. By Lemma 3.4, some $p$-th power of these $\varphi_{i}$ glue into an isomorphism $\varphi: x^{\otimes p^{r}} \stackrel{\sim}{\rightarrow} \mathbb{1}^{\otimes p^{r}}=\mathbb{1}$.

Putting things together, we have our central result:

Theorem 3.9. Let $\mathcal{K}$ be a rigid, idempotent complete, tensor triangulated $\mathbb{Z} / p$ category. Let $U_{*}=\left(U_{i}\right)_{i=1}^{n}$ be an open cover of $\operatorname{Spc}(\mathcal{K})$ as in (10). Let $\sigma_{i j} \in$ $\operatorname{Aut}_{\mathcal{K}\left(U_{i j}\right)}(\mathbb{1})$ be a cocycle: $\sigma_{k j} \cdot \sigma_{j i}=\sigma_{k i}$ on $U_{i j k}$ for all $1 \leq i, j, k \leq n$. Then there exists $r \geq 0$ such that the data $\left(U_{*}, \mathbb{1}_{*}, \sigma_{* *}^{\otimes p^{r}}\right)$ admits a gluing $x(r) \in \mathcal{K}$. Two such gluings $x_{1}\left(r_{1}\right)$ and $x_{2}\left(r_{2}\right)$ have isomorphic $p$-th powers; more precisely, there exists $r_{3} \geq 0$ with $x_{1}\left(r_{1}\right)^{\otimes p^{r_{2}+r_{3}}} \simeq x_{2}\left(r_{2}\right)^{\otimes p^{r_{1}+r_{3}}}$. In particular, the following class in $\operatorname{Pic}_{\text {loc.tr. }}(\mathcal{K}) \otimes_{\mathbb{Z}} \mathbb{Z}[1 / p] \subset \operatorname{Pic}(\mathcal{K}) \otimes_{\mathbb{Z}} \mathbb{Z}[1 / p]$ is independent of the choice of $r$ :

$$
\beta\left(U_{*} ; \sigma_{* *}\right):=[x(r)] \otimes \frac{1}{p^{r}} .
$$

This defines an isomorphism $\beta: \check{\mathrm{H}}^{1}\left(\operatorname{Spc}(\mathcal{K}), \mathbb{G}_{\mathrm{m}}\right) \otimes_{\mathbb{Z}} \mathbb{Z}[1 / p] \stackrel{\sim}{\rightarrow} \operatorname{Pic}_{\text {loc.tr. }}(\mathcal{K}) \otimes_{\mathbb{Z}} \mathbb{Z}[1 / p]$ natural in $\mathcal{K}$ and inverse to the homomorphism $\alpha \otimes \mathrm{id}_{\mathbb{Z}[1 / p]}$ from Construction 2.6.

Proof. The existence of the gluing $x(r)$ is Proposition 3.7. The uniqueness is Proposition 3.6 applied to $x_{1}\left(r_{1}\right)^{\otimes p^{r_{2}}}$ and $x_{2}\left(r_{2}\right)^{\otimes p^{r_{1}}}$ which are two gluings of the same data $\left(U_{*}, \mathbb{1}_{*}, \sigma_{* *}^{\otimes r^{r_{1}+r_{2}}}\right)$. Hence $\beta\left(U_{*}, \sigma_{* *}\right) \in \operatorname{Pic}_{\text {loc.tr. }}(\mathcal{K}) \otimes \mathbb{Z}[1 / p]$ is well defined. 
It is multiplicative in the cocycle by Remark 3.5 and vanishes on boundaries by Proposition 3.8. Hence we have a well-defined homomorphism $\check{\mathrm{H}}^{1}\left(U_{*}, \mathrm{p} \mathbb{G}_{\mathrm{m}}\right) \otimes$ $\mathbb{Z}[1 / p] \longrightarrow \operatorname{Pic}_{\text {loc.tr. }}(\mathcal{K}) \otimes \mathbb{Z}[1 / p]$ for all open covers $U_{*}$ of $\operatorname{Spc}(\mathcal{K})$. This is obviously compatible with the refinement of open covers (a solution of the gluing for $U_{*}$ gives a solution for any refinement). So, we get the announced homomorphism $\beta$. It is straightforward to check that $\beta$ is, by its very construction, a two-sided inverse of $\alpha \otimes \mathrm{id}: \mathrm{Pic}_{\text {loc.tr. }}(\mathcal{K}) \otimes \mathbb{Z}[1 / p] \longrightarrow \check{\mathrm{H}}^{1}\left(\operatorname{Spc}(\mathcal{K}), \mathbb{G}_{\mathrm{m}}\right) \otimes \mathbb{Z}[1 / p]$.

\section{Applications to modular REPRESEntation theOry}

Let $G$ be a finite group and $\mathcal{V}_{G}=\mathcal{V}_{G}(k)=\operatorname{Proj}\left(\mathrm{H}^{\bullet}(G, k)\right)$ its projective support variety over a field $k$, whose characteristic $p$ divides the order of $G$. Let $\mathcal{K}=k G$-stab be the stable category, which has finitely generated $k G$-modules as objects and, as morphisms, $k G$-homomorphisms modulo those which factor via a projective. The tensor product of two $k G$-modules is $M \otimes_{k} N$ with diagonal action of $G$. This $\mathcal{K}$ is a rigid (Definition 1.4 with usual dual $D(M)=M^{*}$ ), idempotent complete (Definition 1.5), $\otimes$-triangulated $\mathbb{Z} / p$-category. See more in Carlson [11] or Benson [9].

A $k G$-module $M$ is $\otimes$-invertible in $k G$-stab (see Definition 2.1) if and only if $M \otimes M^{*} \simeq k$ in $k G-$ stab, which is equivalent to $\operatorname{End}_{k}(M) \simeq k \oplus($ proj $)$ in $k G-\bmod$. The latter has been dubbed endotrivial in the literature. So, the group $T(G)$ of stable isomorphism classes of endotrivial modules is nothing but $\operatorname{Pic}(k G-$ stab).

By [4, Thm.6.3 (b)], we have an isomorphism $\operatorname{Spec}(k G-$ stab $) \simeq \mathcal{V}_{G}(k)$, natural in $G$ (see Remark 4.3), and which is compatible with the support varieties of modules; namely for every finitely generated $k G$-module $M$, we have $\operatorname{supp}(M)=$ $\mathcal{V}_{G}(M)$.

Remark 4.1. At this stage, we can make sense of what should be "the category $k G$-stab on some open $U \subset \mathcal{V}_{G}$ ", by means of triangular geometry; see Section 1 . (Note that $\mathcal{V}_{G}$ is noetherian, so all open subsets are quasi-compact.) The answer is the $\otimes$-triangulated category $\mathcal{K}(U)$ for $\mathcal{K}=k G$-stab, as in Construction 1.9. We also have the restriction functor $\operatorname{res}_{U}: k G-$ stab $\rightarrow \mathcal{K}(U)$. By Remark 1.10, in algebraic geometry the new category $\mathcal{K}(U)$ has the same nature as the original $\mathcal{K}$. This is far from being true in modular representation theory. We shall not use the following result. It is only stated here to emphasize the gain in flexibility provided by the use of tensor triangular geometry in modular representation theory.

Proposition 4.2. Let $U \subsetneq \mathcal{V}_{G}=\operatorname{Spc}(\mathcal{K})$ be a proper, non-empty open and consider $\mathcal{K}(U)$ as in Construction 1.9. Then $\mathcal{K}(U)$ cannot be equivalent to $k G^{\prime}$-stab, as a tensor triangulated category, for any finite group $G^{\prime}$.

Proof. Suppose that $\mathcal{K}(U) \simeq k G^{\prime}$-stab, as a $\otimes$-triangulated category. Then $U=$ $\operatorname{Spc}(\mathcal{K}(U)) \simeq \operatorname{Spc}\left(k G^{\prime}-\operatorname{stab}\right)=\mathcal{V}_{G^{\prime}}=\operatorname{Proj}\left(\mathrm{H}^{\bullet}\left(G^{\prime}, k\right)\right)$ is a projective variety, hence a proper scheme over $k$. Therefore $U$ must be closed in $\mathcal{V}_{G}$, where it is also open. This would imply that $\mathcal{V}_{G}$ is disconnected, which is wrong. (Simply apply Carlson's Theorem, see Theorem [1.7 to the indecomposable $a=\mathbb{1}=k$ whose support is $\mathcal{V}_{G}$.)

Remark 4.3. Given a subgroup $H \subset G$, restriction defines a $\otimes$-triangulated functor $\operatorname{res}_{G, H}: k G$-stab $\rightarrow k H$-stab. This induces a group homomorphism res $\operatorname{res}_{G, H}:$ $T(G) \rightarrow T(H)$ and a morphism of schemes res ${ }_{G, H}^{*}: \mathcal{V}_{H} \rightarrow \mathcal{V}_{G}$. Note finally that 
$\operatorname{Pic}(-)$ is contravariant on schemes, so $\operatorname{res}_{G, H}$ also induces $\operatorname{res}_{G, H}: \operatorname{Pic}\left(\mathcal{V}_{G}\right) \rightarrow$ $\operatorname{Pic}\left(\mathcal{V}_{H}\right)$. This is what we mean here by naturality in the group $G$, not more.

Since here $\operatorname{Pic}\left(\mathcal{V}_{G}\right) \cong \check{\mathrm{H}}^{1}\left(\mathcal{V}_{G}, \mathbb{G}_{\mathrm{m}}\right)$, Construction 2.6 and Theorem 3.9 imply :

Corollary 4.4. There exists a fraction of homomorphisms, natural in $G$ (Remark 4.3),

$$
\operatorname{Pic}\left(\mathcal{V}_{G}\right) \stackrel{\alpha}{\longleftarrow} \operatorname{Pic}_{\text {loc.tr. }}(k G-\text { stab }) \longleftrightarrow T(G),
$$

and $\alpha$ becomes an isomorphism after inverting $p$ on these abelian groups. The resulting natural injective homomorphism $\beta: \operatorname{Pic}\left(\mathcal{V}_{G}\right) \otimes_{\mathbb{Z}} \mathbb{Z}[1 / p] \hookrightarrow T(G) \otimes_{\mathbb{Z}} \mathbb{Z}[1 / p]$ can be described by the gluing of $\mathbb{G}_{\mathrm{m}}$-cocycles as in Section 3 ,

Example 4.5. By the Evens-Venkov Theorem, the $k$-algebra $\mathrm{H}^{\bullet}(G, k)$ is finitely generated. So, there exist $d \geq 1$ and $\zeta_{1}, \ldots, \zeta_{n} \in \mathrm{H}^{d}(G, k)$ such that $\mathcal{V}_{G}=$ $U_{1} \cup \cdots \cup U_{n}$ where $U_{i}:=D\left(\zeta_{i}\right)=\left\{\mathfrak{p} \in \operatorname{Proj}\left(\mathrm{H}^{\bullet}(G, k)\right) \mid \zeta_{i} \notin \mathfrak{p}\right\}$ are principal open subsets of $\mathcal{V}_{G}$. We refer to such $\zeta_{1}, \ldots, \zeta_{n}$ as a system of parameters in $\mathrm{H}^{d}(G, k)$. The cone of each $\zeta_{i}: \Omega^{d}(k) \rightarrow k$ is the suspension of Carlson's $L_{\zeta_{i}}$ module, which has support $V\left(\zeta_{i}\right)=\mathcal{V}_{G} \backslash D\left(\zeta_{i}\right)$; see [9, Prop. II.5.9.1, p. 186]. Hence $\zeta_{i}: \Omega^{d}(k) \rightarrow k$ is an isomorphism on $D\left(\zeta_{i}\right)$ in the sense of triangular geometry; see Construction 1.9. This proves that $\Omega^{d}(k)$ is locally trivial, i.e. defines a class $\left[\Omega^{d}(k)\right] \in \mathrm{Pic}_{\text {loc.tr. }}(\mathcal{K})$; see Definition 2.3. By Construction 2.6, the cocycle $\sigma$ associated to $\left[\Omega^{d}(k)\right]$ under the homomorphism $\alpha: \operatorname{Pic}$ loc.tr. $(\mathcal{K}) \rightarrow \check{\mathrm{H}}^{1}\left(U_{*}, \mathrm{p} \mathbb{G}_{\mathrm{m}}\right)$ is simply $\sigma_{j i}=\zeta_{j} \circ \zeta_{i}^{-1} \in \operatorname{Aut}_{\mathcal{K}\left(U_{i j}\right)}(\mathbb{1})$. On the other hand, it is easy to check that this class $[\sigma] \in \check{\mathrm{H}}^{1}\left(\mathcal{V}_{G}, \mathrm{p} \mathbb{G}_{\mathrm{m}}\right) \cong \operatorname{Pic}\left(\mathcal{V}_{G}\right)$ corresponds to the line bundle $\mathcal{O}_{\mathcal{V}_{G}}(-d)$ over $\mathcal{V}_{G}$. Indeed, each $\zeta_{i} \in \mathrm{H}^{d}(G, k)$ defines a global homomorphism $\mathcal{O}_{\mathcal{V}_{G}}(-d) \rightarrow \mathcal{O}_{\mathcal{V}_{G}}$ (see Hartshorne [18, § II.5, p. 116]) which is an isomorphism on $U_{i}=D\left(\zeta_{i}\right)$. We have the commutative diagram

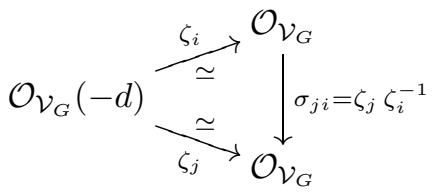

of sheaves on $U_{i j}=D\left(\zeta_{i} \zeta_{j}\right)$, analogous to (9) in $\mathcal{K}\left(U_{i j}\right)$. This shows that the sheaf $\mathcal{O}_{\mathcal{V}_{G}}(-d)$ is the gluing corresponding to our cocycle $\sigma$ as announced; see [18, Ex. II.1.22]. This proves that $\alpha\left(\left[\Omega^{d}(k)\right]\right)=\left[\mathcal{O}_{\mathcal{V}_{G}}(-d)\right]$ for that specific integer $d \geq 1$. Hence, computing in $T(G) \otimes \mathbb{Q}$, we obtain

$\left(\beta \otimes \mathrm{id}_{\mathbb{Q}}\right)\left(\left[\mathcal{O}_{\mathcal{V}_{G}}(-d)\right] \otimes \frac{1}{d}\right)=\left(\alpha \otimes \operatorname{id}_{\mathbb{Q}}\right)^{-1}\left(\left[\mathcal{O}_{\mathcal{V}_{G}}(-d)\right] \otimes \frac{1}{d}\right)=\left[\Omega^{d}(k)\right] \otimes \frac{1}{d}=\left[\Omega^{1}(k)\right] \otimes 1$.

Remark 4.6. Note that the $\mathcal{O}_{\mathcal{V}_{G}}$-module $\mathcal{O}_{\mathcal{V}_{G}}(m)$ is not necessarily a line bundle for all $m \in \mathbb{Z}$. This comes from $\mathrm{H}^{\bullet}(G, k)$ not being generated by $\mathrm{H}^{1}(G, k)$. Therefore, we cannot replace the above $\mathcal{O}_{\mathcal{V}_{G}}(-d) \otimes \frac{1}{d}$ by $\mathcal{O}_{\mathcal{V}_{G}}(-1) \otimes 1$, a priori.

Theorem 4.7. Let $G$ be a finite group and let $k$ be a field of characteristic $p$. Then the gluing map $\beta$ of Corollary 4.4 induces an isomorphism, natural in $G$ (see Remark 4.3),

$$
\beta: \operatorname{Pic}\left(\mathcal{V}_{G}\right) \otimes \mathbb{Q} \stackrel{\sim}{\longrightarrow} T(G) \otimes \mathbb{Q} .
$$


Moreover, this isomorphism maps $\left[\mathcal{O}_{\mathcal{V}_{G}}(-d)\right] \otimes \frac{1}{d}$ to $[\Omega(k)] \otimes 1$ for every $d \geq 1$ such that $\mathrm{H}^{d}(G, k)$ contains a system of parameters (see Example 4.5).

Proof. By Corollary 4.4 it suffices to prove that the subgroup Pic loc.tr. $(k G-$ stab) has finite index in $T(G)$. In [6, $\S 10]$, we proved that $T(G)$ admits a subgroup of finite index generated by endotrivial modules $X$ which are the result of gluing two copies of $k$ over a cover of $\mathcal{V}_{G}$ by two open pieces. These modules $X$ belong to the subgroup Pic loc.tr. $(k G-$ stab) by definition (Definition 2.3), hence the result.

Remark 4.8. For experts, we outline the construction, given in [], of enough locally trivial modules to prove that Pic loc.tr. $(k G$-stab) has finite index in $T(G)$. When $G$ has $p$-rank one, $T(G)$ is finite and the result is obvious. So let us assume that $G$ has $p$-rank at least two. Then there exist elementary abelian $p$-subgroups $E_{1}, \ldots, E_{n} \subset$ $G$ of $p$-rank two, such that:

(a) when $G$ has $p$-rank two, $E_{1}, \ldots, E_{n}$ form a complete set of representatives of the conjugacy classes of maximal elementary abelian $p$-subgroups;

(b) when $G$ has $p$-rank three or more, $E_{1}, \ldots, E_{n-1}$ form a complete set of representatives of the conjugacy classes of maximal elementary abelian $p$ subgroups of $p$-rank two and every maximal elementary abelian $p$-subgroup of $p$-rank three or more contains a conjugate of $E_{n}$.

The homomorphism $\rho: T(G) \rightarrow \prod_{i=1}^{n} T\left(E_{i}\right) \simeq \mathbb{Z}^{n}$ has finite kernel. (This $n$ will be the dimension of $T(G) \otimes \mathbb{Q}$.) It now suffices to prove that for each $i=1, \ldots, n$ we can produce an endotrivial module $M_{i} \in \operatorname{Pic}_{\text {loc.tr. }}(k G$-stab) whose image under $\rho$ is $\left(k, \ldots, k, \Omega^{r_{i}}(k), k \ldots, k\right)$ in $\prod_{i=1}^{n} T\left(E_{i}\right)$ for some $r_{i} \neq 0$. Moreover, by Example 4.5. we have $\Omega^{d}(k) \in \operatorname{Pic}_{\text {loc.tr. }}(k G$-stab) for some $d>0$, whose image under $\rho$ is $\left(\Omega^{d}(k), \ldots, \Omega^{d}(k)\right)$, so it suffices to produce the above $M_{i}$ only for $i=1, \ldots, n-1$ and we can assume $i=1$. Then, using Quillen's Theorem, one shows the existence of two elements $\eta, \zeta \in \mathrm{H}^{r_{1}}(G, k)$ for some $r_{1}>0$ such that $\eta$ restricts to zero on every maximal elementary abelian $p$-subgroup not conjugate to $E_{1}$ and such that $\eta, \zeta$ restrict to a system of parameters in $\mathrm{H}^{\bullet}\left(E_{1}, k\right)$ (see Example 4.5). One then considers the disjoint closed subsets $W_{1}=V(\zeta) \cap \operatorname{res}_{G, E_{1}}^{*}\left(\mathcal{V}_{E_{1}}\right)$ and $W_{2}=$ $V(\eta)$. Their open complements $\left(U_{i}:=\mathcal{V}_{G} \backslash W_{i}\right)_{i=1,2}$ form an open cover of $\mathcal{V}_{G}=$ $\operatorname{Spc}(k G-$ stab $)$. The support of the cone of $\eta: \Omega^{r_{1}}(k) \rightarrow k$ is exactly $W_{2}$; hence $\eta$ is an isomorphism on $U_{2}$ and a fortiori on $U_{1} \cap U_{2}$. Moreover, by construction of $\eta, V(\eta)=W_{2}$ contains all irreducible components of $\mathcal{V}_{G}$ except $\operatorname{res}_{G, E_{1}}^{*}\left(\mathcal{V}_{E_{1}}\right)$. So, $V(\zeta) \subset W_{1} \cup W_{2}$, meaning that $\zeta: \Omega^{r_{1}}(k) \rightarrow k$ is an isomorphism on $U_{1} \cap U_{2}$. In short, we have an isomorphism

$$
k \stackrel{\zeta}{\longleftarrow} \Omega^{r_{1}}(k) \stackrel{\eta}{\longrightarrow} k
$$

on $U_{1} \cap U_{2}$ that we can use to glue two copies of $k$, that is, $x_{i}=k$ on $U_{i}$ for $i=1,2$, along the isomorphism $\sigma_{21}=\eta \circ \zeta^{-1}$ on $U_{1} \cap U_{2}$; see Theorem 2.8. We proved in [6. Thm. 10.2] that the resulting gluing in $k G$-stab is the wanted endotrivial module $M_{1}$; that is, its image under $\rho$ is $\left(\Omega^{r_{1}}(k), k, \ldots, k\right)$.

Example 4.9. Theorem 4.7 does not hold integrally. Take for instance $G=Q_{8}$ the quaternion group of order 8 and $p=2$. Then $G$ has 2-rank one, hence $\mathcal{V}_{G}$ is a point and $\operatorname{Pic}\left(\mathcal{V}_{G}\right)=0$, but $T(G)=\mathbb{Z} / 4$ when $k$ contains no cubic root of unity and $T(G)=\mathbb{Z} / 4 \oplus \mathbb{Z} / 2$ otherwise. See Dade [17] or Carlson-Thévenaz [14, Thm. 6.3]. 
We end the paper with an obvious application of Theorem 4.7:

Corollary 4.10. Let $H \subset G$ be a subgroup inducing an isomorphism $\mathcal{V}_{H} \stackrel{\sim}{\rightarrow} \mathcal{V}_{G}$ on support varieties (e.g. $H$ controls p-fusion; see Mislin [21] or Alperin [2]). Then $\operatorname{res}_{G, H} \otimes \operatorname{id}_{\mathbb{Q}}: T(G) \otimes \mathbb{Q} \stackrel{\sim}{\rightarrow} T(H) \otimes \mathbb{Q}$ is an isomorphism.

Example 4.11. Corollary 4.10 does not hold integrally either. Let $p>2$ be an odd prime and let $n$ be an integer $2 p \leq n<p^{2}$. Let $G$ be the symmetric group on $n$ letters. As explained in Carlson-Mazza-Nakano [13, Thm. A (c)], we have $T(G) \simeq \mathbb{Z} \oplus(\mathbb{Z} / 2)^{2}$ when $2 p \leq n<3 p$ and $T(G) \simeq \mathbb{Z} \oplus \mathbb{Z} / 2$ when $3 p \leq n<p^{2}$. On the other hand, a $p$-Sylow subgroup $P \subset G$ is abelian (even elementary abelian of $p$-rank $\left.\left[\frac{n}{p}\right]\right)$. So, by a celebrated theorem of Burnside, its normalizer $H:=N_{G}(P)$ controls $p$-fusion. Yet, the torsion part of $T(H)$ is $\mathbb{Z} /(p-1) \oplus \mathbb{Z} / 2$ by [13, $\S 6$.

\section{ACKNOWLEDGMENTS}

The author wishes to thank Dave Benson, Jon Carlson, Eric Friedlander, Nadia

Mazza, Julia Pevtsova and Jacques Thévenaz for precious comments and numerous references.

\section{REFERENCES}

1. J. Alperin. A construction of endo-permutation modules. J. Group Theory, 4(1):3-10, 2001. MR:1808834 (2001m:20012b)

2. J. Alperin. On a theorem of Mislin. J. Pure Appl. Algebra, 206(1-2):55-58, 2006. MR2220080 (2007a:20052)

3. P. Balmer. Presheaves of triangulated categories and reconstruction of schemes. Math. Ann., 324(3):557-580, 2002. MR 1938458 (2003j:18016)

4. P. Balmer. The spectrum of prime ideals in tensor triangulated categories. J. Reine Angew. Math., 588:149-168, 2005. MR2196732 (2007b:18012)

5. P. Balmer. Supports and filtrations in algebraic geometry and modular representation theory. Amer. J. Math., 129(5):1227-1250, 2007. MR2354319

6. P. Balmer, D. J. Benson, and J. F. Carlson. Gluing representations via idempotent modules and constructing endotrivial modules. J. Pure Appl. Algebra, 213(2):173-193, 2009.

7. P. Balmer and G. Favi. Gluing techniques in triangular geometry. Q. J. Math., 51(4):415-441, 2007. MR2371464 (2008m:18020)

8. P. Balmer and M. Schlichting. Idempotent completion of triangulated categories. J. Algebra, 236(2):819-834, 2001. MR1813503 (2002a:18013)

9. D. J. Benson. Representations and cohomology I $\mathscr{G}$ II, volume $30 \& 31$ of Cambridge Studies in Advanced Mathematics. Cambridge University Press, 1998. MR.1644252 (99f:20001a) MR.1644252 (99f:2001b)

10. A. B. Buan, H. Krause, and $\varnothing$. Solberg. Support varieties: an ideal approach. Homology, Homotopy Appl., 9(1):45-74 (electronic), 2007. MR2280286 (2008i:18007)

11. J. F. Carlson. Modules and group algebras. Lectures in Mathematics ETH Zürich. Birkhäuser Verlag, Basel, 1996. MR:1393196 (97c:20013)

12. J. F. Carlson. Constructing endotrivial modules. J. Pure Appl. Algebra, 206(1-2):83-110, 2006. MR.2220083 (2006m:20017)

13. J. F. Carlson, N. Mazza, and D. Nakano. Endotrivial modules for the symmetric and alternating groups. Proc. Edinburgh Math. Soc. (2), 52(1):45-66. MR2475880

14. J. F. Carlson and J. Thévenaz. Torsion endo-trivial modules. Algebr. Represent. Theory, 3(4):303-335, 2000. MR1808129 (2001m:20014)

15. J. F. Carlson and J. Thévenaz. The classification of endo-trivial modules. Invent. Math., 158(2):389-411, 2004. MR2096798 (2005e:20013)

16. J. F. Carlson and J. Thévenaz. The classification of torsion endo-trivial modules. Ann. of Math. (2), 162(2):823-883, 2005. MR2183283(2006f:20012)

17. E. C. Dade. Une extension de la théorie de Hall et Higman. J. Algebra, 20:570-609, 1972. MR0297888 (45:6940) 
18. R. Hartshorne. Algebraic geometry. Springer-Verlag, New York, 1977. Graduate Texts in Mathematics, No. 52. MR0463157 (57:3116)

19. B. Keller and A. Neeman. The connection between May's axioms for a triangulated tensor product and Happel's description of the derived category of the quiver D4. Doc. Math., 7:535560, 2002. MR2015053 (2004j:18007)

20. S. Mac Lane. Categories for the working mathematician, volume 5 of Graduate Texts in Mathematics. Springer-Verlag, New York, 1998. MR1712872 (2001j:18001)

21. G. Mislin. On group homomorphisms inducing mod- $p$ cohomology isomorphisms. Comment. Math. Helv., 65(3):454-461, 1990. MR 1069820 (92a:20059)

22. R. W. Thomason and T. Trobaugh. Higher algebraic $K$-theory of schemes and of derived categories. In The Grothendieck Festschrift, Vol. III, volume 88 of Progr. Math., pages 247435. Birkhäuser, Boston, MA, 1990. MR.1106918 (92f:19001)

23. J.-L. Verdier. Des catégories dérivées des catégories abéliennes. Astérisque, 239:xii+253 pp. (1997), 1996. MR 1453167 (98c:18007)

Department of Mathematics, University of California, los Angeles, Los Angeles, CALifornia 90095-1555

E-mail address: balmer@math.ucla.edu

$U R L:$ http://www.math.ucla.edu/ balmer 